\title{
Analytical methodology to elemental quantification of weathered terrestrial analogues to meteorites using a portable Laser-Induced Breakdown Spectroscopy (LIBS) instrument and Partial Least Squares (PLS) as multivariate calibration technique
}

Leticia Gómez-Nubla*, Julene Aramendia, Silvia Fdez-Ortiz de Vallejuelo*, Juan Manuel Madariaga

Department of Analytical Chemistry, Faculty of Science and Technology, University of the Basque Country UPV/EHU, P.O. Box 644, E-48080 Bilbao, Basque Country, Spain *Corresponding author: Tel.:+34 9460182 97; Fax: +34 9460135 00; E-mail address: leticia.gomez@ehu.eus (Leticia Gómez-Nubla) silvia.fernandez@ehu.eus (Silvia Fdez-Ortiz de Vallejuelo)

\begin{abstract}
The present work is focused on the in situ quantitative analysis of $\mathrm{Si}, \mathrm{Al}, \mathrm{Mg}, \mathrm{Ca}, \mathrm{Ba}, \mathrm{Na}$, and $\mathrm{Fe}$, present in weathered terrestrial analogues to meteorites (black steel slag and impact glasses), using a portable Laser Induced Breakdown Spectroscopy (LIBS) instrument. For that purpose, several standards pellets of known elemental concentrations were manufactured. The elemental and molecular homogeneity of the pellets was studied by means of Scanning Electron Microscopy coupled to Energy Dispersive X-ray spectroscopy (SEM-EDS) and Raman spectroscopy. This check was always made before the LIBS analysis. Univariate and multivariate (Partial Least Squares (PLS) regression) calibration approaches on LIBS spectra were selected as initial calibration models. After a comparison between both approaches, the former was discarded due to the poor linearity of the calibration curves, and PLS regression was chosen to treat the LIBS spectra as the multivariate calibration approach (in the ultraviolet (UV) and infrared (IR) spectral ranges). Predictive capabilities of each calibration model were evaluated by calculating regression coefficient (r), number of PLS factors (rank), relative errors of cross validation (RMSECV), residual predictive deviation (RPD) and the Bias value. At the end, the simultaneous use of both ranges of wavelengths was demonstrated to be more fruitful rather than using the individual ones, probably due to the higher number of emission lines, number of spectral variables and the PLS latent variables for each element. Moreover, a Reference Material was used as external validation, obtaining satisfactory results in the determination of elements. The predictive ability of the PLS models was evaluated on samples of Darwin Glasses (Australia), Libyan Desert Glasses (Western Desert of Egypt) and black steel slag residues (steelworks of Basque Country). The obtained results were in concordance with the range of composition measured also by X-ray Fluorescence Spectrometer (ED-XRF). Our
\end{abstract}


methodology is a good, rapid, simple and cost-effective alternative for in situ analysis of these terrestrial analogues over other techniques.

Keywords: terrestrial analogues to meteorites; black steel slag; Libyan Desert Glass; Darwin Glass; portable LIBS; Partial Least Square regression.

\section{Introduction}

Laser-Induced Breakdown Spectroscopy (LIBS) is a spectroscopic multi-elemental analytical technique based on Atomic Emission Spectroscopy (AES). The elements included in the sample are vaporized, atomized and excited in hot plasma generating an atomic and ionic spectrum that is characteristic of the elemental composition of the sample [1]. LIBS has been widely employed in the chemical analysis of meteorites of diverse nature using different laboratory based set-ups [2-5]. Stand-off LIBS at distances up to $8.3 \mathrm{~m}$ was used by Thompson et al. in 2006 [6] to analyse two Martian meteorites, Zagami and Dar al Gani 476, with the aim to check laboratory configurations similar to the ones designed for the ChemCam instrument on-board of the Curiosity rover sent to Mars in 2011. De Giacomo et al. used in 2007 a laboratory benchtop set up at $90^{\circ}$ between the pulsed laser impacting in the sample and the collection of the emission lines, to analyse four different meteorites: Dofhar 461 (lunar meteorite), Chondrite L6 (stony meteorite), Dofhar 019 (Mars meteorite) and Sikhote Alin (irony meteorite) [7]. The same authors used the same configuration to perform in 2010 the first concentration profile (50 $\mu \mathrm{m}$ distance between two consecutive spots) of $\mathrm{Ni}$ and Co along the Widmanstätten structure on a sample of Toluca meteorite [8]. Analytical applications of LIBS in the field of space, as well as environment and cultural heritage, were reviewed in 2010 pointing out the excellent spatial resolution thanks to the micrometric laser spot [9].

The LIBS applications to the study of geomaterials, including weathering and alteration of minerals in meteorites and planetary analogues, was reviewed in 2014 [1]. In the same year the first review on portable LIBS was published [10]. Since then, several works using portable devices have been published to characterize different types of geomaterials including particulate matter in suspended sediments [11].

To carry out the quantitative analysis of solid samples, there are two traditional approaches for calibration: a calibration free procedure (CF-LIBS) and a calibration line approach $[12,13]$. CFLIBS determines the elemental composition of samples directly from measured LIBS spectra using computational methods in analyzing the basic physics of the plasma process. The plasma temperature and electron number density are estimated assuming that the plasma composition represents exactly the composition of the sample, i.e. the conditions of stoichiometric ablation 
are obeyed [14]. This quantitative procedure has been applied to the analysis of found meteorite fragments [3] and even to classify them after the quantification of the elements present in the analyzed meteorite [5].

The calibration line approach consists in drawing a calibration line with a set of standards whose composition is similar to that of the unknown sample. The intensity or area of a given emission line is proportional to the number density of emitters, which, in turn, is proportional to the concentration of the emitter (namely, the analyte) in the irradiated sample. Therefore, the emission intensity is linearly correlated to the concentration of the given element in the sample [15], thus it is feasible to determine unknown concentrations of elements in the sample by comparison with standard samples of known concentrations.

The usual calibration line approach belongs to the univariate calibration methods that use a single characteristic spectral line of the measured element as an input variable to set up a univariate regression model [16]. However, due to the complex spectra of multi-component samples and the overlapping of spectral lines, univariate calibrations can produce biased concentration values. Therefore, multivariate calibrations are recommended to be employed in order to improve the accuracy of the quantitative analysis [17].

Multivariate calibration techniques such as partial least squares regression (PLS), principal component regression (PCR), multi-linear regression (MLR) or artificial neural networks (ANN) can be used [18]. In general, partial least squares regressions (PLS) are the most employed among the multivariate calibration techniques applied to measure the concentrations of elements by LIBS [19-20]. Such methods display the concentration of elements taking into account all the variables belonging to the spectrum, reducing the complexity of spectra and enabling to extract the valuable information. Moreover, other chemometric methods such as Principal Components Analysis (PCA) are often coupled to calibration techniques in order to observe data clusters and discriminate outliers, prior to performing the current quantitative determination.

The LIBS research on terrestrial analogues to extraterrestrial materials, in laboratory conditions or in situ with portable equipment, will be useful to improve and develop future experimental strategies of extraterrestrial exploration without destroying extraterrestrial samples. In this sense, the methodology adopted to validate the developed Raman spectroscopy procedures, in the Exomars2020 mission to Mars, will use Martian meteorites previously characterised. These Martian meteorites are scarce and sample consumption is not recommendable, thus terrestrial analogues should be used to optimize procedures for elemental identification and quantification that will be applied in the near future to extraterrestial materials.

In the search for terrestrial analogues to meteorites, we have noticed that several studies have found similarities with respect to their composition between meteorites and black slag by- 
products or some impact glasses, which could be due to their silica matrix and the high temperature of their formation [21-24]. On one side, black slags are residues come from the steel production that have suffered temperature gradients up to $1000^{\circ} \mathrm{C}$. On the other side, impact glasses like Libyan Desert Glass (LDG) and Darwin Glass (DG) are terrestrialextraterrestrial materials generated after the impact of a low airburst or hypervelocity extraterrestrial bolide on Earth. In this case, LDG was found in the desert of eastern Libya and western Egypt, and DG in south of Queenstown in West Coast of Tasmania. Our mineralogical studies on such black slags [25] and LDG and DG [26, 27] suggest that could be considered terrestrial analogues to meteorites. Besides, these materials in open air can also suffer the same alteration processes that affect to meteorites on the surface of the Earth.

The main aim of our work was to develop a fast, cheap, and effective methodology for the quantitative analysis of these weathered terrestrial analogues to meteorites using commercial portable LIBS equipment that allows us to carry out in situ measurements. To guarantee the required homogeneity at the spot level (around 200 microns in diameter), Electron Microscopy/Energy Dispersive X-Ray Spectroscopy (SEM-EDS) and Raman spectroscopy were employed to study the elemental and molecular distribution of the standards prior to performing any LIBS analysis (techniques already employed in the analysis of meteorites [28]) Moreover, Micro X-ray Fluorescence ( $\mu$-XRF) analysis was used to corroborate the predicted concentration of the slag and impact glass samples. The final goal was to prepare a routine method without sample preparation steps, and negligible influence of operators on analytical results.

\section{Material and methods}

\subsection{Material}

19 pellets were prepared as multi-component oxide materials of $\mathrm{SiO}$ (Alfa Aesar, 99.5\%), $\mathrm{MgO}$ (Sigma-Aldrich, >99\%), $\mathrm{CaO}$ (Sigma-Aldrich, 99.9\%), $\mathrm{Al}_{2} \mathrm{O}_{3}$ (Sigma-Aldrich, 99.99\%), $\mathrm{BaO}$ (Sigma-Aldrich, 99.99\%), $\mathrm{Na}_{2} \mathrm{O}+\mathrm{Na}_{2} \mathrm{O}_{2}$ (Alfa Aesar, 99\%) and $\mathrm{Fe}_{2} \mathrm{O}_{3}$ (Alfa Aesar, 99.99\%) powders in order to perform a calibration line. The concentration ranges of each element varied according to the percentages that could be found in the terrestrial analogues to meteorites samples (see Fig. 1). The total weight of each powder mixture was around $0.50 \mathrm{~g}$ (analytical balance with $\pm 0.0001 \mathrm{~g}$ of instrumental precision). No compound was used as a binder.

The mixture of powders was milled in an agate mortar to ensure the homogeneity, and after, were hydraulically pressed at 9 Ton. $\mathrm{cm}^{-1}$ of pressure (CrushIR, PIKE Technologies, Canada). The final pellets had a diameter of $12 \mathrm{~mm}$ and approximately $1 \mathrm{~mm}$ of thickness. In the same way, a pellet for each standard (oxide powders) was prepared with the purpose to select the appropriate lines in the LIBS spectra for each element taking into account the sensitivity of our 
LIBS system. Afterward, these pellets were immediately stored in zip bags, without atmosphere contact, otherwise their volume could increase in a short time with high humidity levels due to hydration of $\mathrm{CaO}$ and $\mathrm{MgO}$ and can be easily broken.

The weathered terrestrial-extraterrestrial samples to analyze were black steel slags from three open air storage place of different steel productions (namely common steel, special steel and stain less steel industries), and 7 impact glasses from the Meteorites Collection of the Basque Country University (UPV/EHU), of which 3 are LDGs and 4 are DGs.

Moreover, to perform the external validation, 6 pellets were prepared mixing a steelmaking slag Reference Material (RM, NH-146, Brammer standard, Mittal Steel Oslava, Czech Republic) with ethylcellulose. It was obtained different known concentrations of $\mathrm{Si}, \mathrm{Mg}, \mathrm{Ca}, \mathrm{Al}$, and $\mathrm{Fe}$, within the concentration range of the calibration pellets.

\subsection{Methods}

2.2.1 Scanning Electron Microscopy coupled to Energy Dispersive X-Ray Spectroscopy (SEM$E D S)$

In order to know the elemental homogeneity of the pellets, a JEOL JSM-6400 SEM with an Oxford Pentafet photon energy instruments Link Isis X-Ray (EDS) system was used. SEM images were acquired at high vacuum employing an acceleration voltage of $20 \mathrm{KV}$ as well as in the elemental mapping. Magnifications up to $10000 \times$ were reached using a Secondary Electron (SE) detector for image acquisitions, and elemental mappings were performed using a $15 \mathrm{~mm}$ working distance. No pretreatment was applied since the samples were considered to be conductive enough due to their iron content. The analysis and the treatment of the data were performed with the INCA Microanalysis Suite 4.3 (Oxford Instruments, UK).

\subsubsection{Raman spectroscopy}

The molecular homogeneity of the pellets was studied by Raman spectroscopy. For that, a Renishaw InVia confocal micro Raman spectrometer (Renishaw, UK) provided by a $514 \mathrm{~nm}$ lasers as excitation sources (at highest powers, the nominal laser powers at the source is $50 \mathrm{~mW}$ and at the samples $20 \mathrm{~mW}$ ) was used. This equipment is coupled to a Leica DMLM microscope (Bradford, UK). For visualization and focusing 5x N PLAN (0.12 aperture) and 20x N PLAN EPI ( 0.40 aperture) lenses were used. The spectra were acquired using 50x N PLAN (0.75 aperture, lateral resolution of $2 \mu \mathrm{m}$ ) long-range objectives. The microscope implements a Prior Scientific motorized XYZ positioning stage with a joystick and is equipped with a microcamera for searching points of interest. Moreover, the equipment is installed on an antivibratory table inside a temperature-controlled chamber. 
Apart from detailed measurements, this equipment was used to obtain Raman chemical image acquisitions of specific areas of the samples (the measured area in each sample was of $70 \times 70$ $\mu \mathrm{m}$ ) by means of the Stream Line mode (the sample is moved under the lens, to be the line raster across the area of interest), outlining the distribution of several chemical components in those areas. A great quantity of spectra and processing images were collected, creating falsecolour Raman chemical imaging to perform the molecular homogeneity analyses.

The spectral range was $108-2000 \mathrm{~cm}^{-1}$ with a resolution of $1 \mathrm{~cm}^{-1}$. In the detailed measurements, the number of accumulations and integration time were varied in order to achieve the best signal-to-noise ratio. For the Raman images, the acquisition time was $5 \mathrm{~s}$ with one accumulation per each spectrum, which ensured a suitable signal-to-noise ratio, and the step size was set in $3.2 \mu \mathrm{m}$. Finally, around 132 spectra were obtained. WIRE 3.2 software (Renishaw, UK) was used to the data obtaining and their analysis and treatment was carried out with the Omnic software. In the data collected for each map, a cosmic ray removal and a noise filter were performed. After accomplishing the baseline correction, the spectra were filtered based on the integration of the main Raman bands for each molecular phase in order to represent their chemical image in the selected mapping area. In case of mineral phases sharing band positions, secondary Raman bands were taken into account for peak integration.

\subsubsection{Laser-Induced Breakdown Spectroscopy (LIBS)}

A commercial portable EasyLIBS instrument (IVEA system, model Easy 2C, Orleans, France) was employed for these measurements. It uses a pulsed Nd:YAG laser, with the possibility to dual pulse mode, and emits at the fundamental wavelength of $1064 \mathrm{~nm}$. The laser energy per pulse on the sample is higher than $25 \mathrm{~mJ}$ with a repetition rate of $1 \mathrm{~Hz}$ and the duration of laser pulse is 4-5 ns. In this work, the measurements were performed with the double pulse mode. An optimized delay time of $50 \mu$ s (both single to double pulse) to the laser pulse and a gate width of $5 \mathrm{~ms}$ were employed. All spectra were accumulated over 1-3 laser-shots.

The Easy $2 \mathrm{C}$ model consists of an optic probe in form of gun that allows focusing the laser in the material to analyze. The gun goes connected to a computer and two spectrometers corresponding to: Ultraviolet (UV) (196-419 nm) and near Infrared (NIR) (575-1000 nm) spectral range (Czerny-Turner Ocean Optics HR 2000+). Both spectral ranges were covered in each acquisition process. The spectral resolution in both is $0.2 \mathrm{~nm}$ (Full Width at Half Maximum, FWHM). The laser spot is around $193 \mu \mathrm{m}$ in diameter and the crater is about 250 $\mu \mathrm{m}$. The plasma light generated by the laser shot is collected with an aspheric lens (from Ocean Optics), directly coupled to a transport fiber. We assumed that all the molecules are dissociated into the LIBS plasma due to the high temperatures generated $(\mathrm{T}>10000 \mathrm{~K})$. A Charge Coupled Device (CCD) detector collects the signal. 
Three measurements per sample were performed in different positions of its surface, aprox. each $4 \mathrm{~mm}$ in the pellets and aprox. each $5 \mathrm{~mm}$ in the weathered terrestrial-extraterrestrial samples, depending on the superficial irregularities. At each position, the experimental procedure firstly started in firing 4 cleaning shots, in order to avoid detecting contaminations at the surface of the pellets or due to possible impurities on the surface of the terrestrial analogues to meteorites.

The software used for automatic acquisition, control, visualization and processing of the spectra was the AnaLIBS version 6.3 from IVEA. It also incorporates two databases (IVEA database and NIST database of atomic spectral data. http://physics.nist.gov/PhysRefData/ASD) to aid in the automatic detection mode and the association of spectral lines to their corresponding chemical elements.

The statistic treatment of the data was performed by means of Principal Component Analysis (PCA) using MATLAB 2010 with PLS Toolbox, version 7.0.2 (Eigenvector Technologies) and OPUS program Version 7.2 from Bruker Gmbh (Bremen, Germany) was used to develop PLS models for elemental quantification.

\subsubsection{Micro-Energy Dispersive X-Ray Fluorescence Spectroscopy}

The M4 TORNADO micro energy dispersive X-ray fluorescence ( $\mu$-ED-XRF) spectrometer (Bruker Nano GmbH, Berlin, Germany) was used to the semiquantitative analysis of the ten terrestrial analogues to meteorites. The equipment implements an Rh tube powered by a lowpower high voltage generator and cooled by air. This tube can operate at voltages in the range of $10-50 \mathrm{kV}$ and currents in the range of $100-700 \mu \mathrm{A}$. It is mounted on a mechanical collimator that allows performing measurements under a lateral/spatial resolution (spot) of $1 \mathrm{~mm}$. An energy-dispersive SDD detector with $30 \mathrm{~mm}^{2}$ sensitive area and energy resolution of $142 \mathrm{eV}$ for $\mathrm{Mn} \mathrm{K}_{\alpha}$ line performs the detection of the fluorescence radiation emitted by the elements on the sample. Five repetitive punctual measurements in several points (aprox. each $5 \mathrm{~mm}$ depending on the superficial irregularities) of each sample were performed. The measuring time (Live Time) used for each punctual measurement was $15 \mathrm{~s}$. Once the spectra were acquired, semiquantitative information was obtained from them. M-Quant quantification software package based on the use of Fundamental Parameters and included in the instrument was employed to the semiquantitative results.

\section{Results and discussion}

\subsection{Homogeneity of the powder pellets}


The homogeneity is an important factor in the case of calibration samples. Therefore, Scanning Electron Microscopy coupled to Energy Dispersive X-ray spectroscopy (SEM-EDS) and Raman imaging were used to examine the elemental and molecular distribution respectively.

\subsubsection{Scanning Electron Microscopy- Energy Dispersive X-ray Spectroscopy (SEM-EDS)}

The SEM images showed that the size of the grains in the calibration samples was between 1-5 $\mu \mathrm{m}$ range (see Fig. $2 \mathrm{i}$ as an example). If we take into account the diameter of the spot analysed by the portable LIBS instrument (around $200 \mu \mathrm{m}$ ), a high number of individual crystals/grains are ablated per shot. Moreover, after obtaining the elemental distribution by EDS mapping of the samples, cameo images over SEM images were performed, and homogeneous elemental distribution was determined (see Fig. 2ii).

\subsubsection{Raman spectroscopy imaging}

During the Raman analysis of all the oxide mixtures in the calibration pellets, it was observed that $\mathrm{Al}_{2} \mathrm{O}_{3}$ did not gave a Raman response, which is due to some polymorphs of alumina do not give Raman signal. Likewise, $\mathrm{CaO}$ and $\mathrm{MgO}$ are also inactive in Raman spectroscopy [29], so they could not be identified. $\mathrm{BaO}$ was not possible to detect either. Then, we were only able to identify $\mathrm{Fe}_{2} \mathrm{O}_{3}$ (Raman bands at 223s, 288vs, 408m, 497w and 608w cm $\mathrm{w}^{-1}$ ), $\mathrm{SiO}$ (Raman band at $460 \mathrm{br} \mathrm{cm}{ }^{-1}$ ) and $\mathrm{Na}_{2} \mathrm{O}+\mathrm{Na}_{2} \mathrm{O}_{2}$ (Raman bands at 236vs, $735 \mathrm{~m}, 790 \mathrm{w}$ and $1077 \mathrm{~s} \mathrm{~cm}^{-1}$ ). Finally, by means of Raman imaging, the homogeneous distribution was observed for those active oxides in Raman (see Fig. 2iii).

On the other hand, we recognized that, after some hours in contact with the atmosphere, some new compounds were formed in the pellets, such as carbonates (calcite $\left(\mathrm{CaCO}_{3}\right.$, Raman bands at $155 \mathrm{~m}, 281 \mathrm{~m}, 712 \mathrm{~m}, 1085 \mathrm{vs}, 1433 \mathrm{w}$ and $\left.1747 \mathrm{w} \mathrm{cm} \mathrm{cm}^{-1}\right)$ and witherite $\left(\mathrm{BaCO}_{3}\right.$, Raman bands at $688 \mathrm{~m}, 1059 \mathrm{~s}$ and $\left.1418 \mathrm{w} \mathrm{cm}^{-1}\right)$ ), and hydroxides (brucite $\left(\mathrm{Mg}(\mathrm{OH})_{2}\right.$, Raman bands at $278 \mathrm{~m}$ and $\left.443 \mathrm{~m} \mathrm{~cm}^{-1}\right)$ and portlandite $\left(\mathrm{Ca}(\mathrm{OH})_{2}\right.$, Raman band at $\left.\left.356 \mathrm{~s} \mathrm{~cm}^{-1}\right)\right)$. Besides, after one week, Raman bands related to $\mathrm{H}_{2} \mathrm{O}$ were also identified in the calibration pellets (Raman bands at $\approx 3000-3600 \mathrm{~cm}^{-1}$ [30]). It was concluded that the contact with the atmosphere must be avoided after preparation of the standards to avoid systematic sources of errors in the quantitative analysis. Hence, once we made new pellets, they were stored in zip bags until their LIBS analysis.

\subsection{LIBS analysis of the powder pellets}

\subsubsection{Identification of outliers}


The identification of outliers between replicates of the calibration data set (19 calibration samples x 3 replicates, 57 total analyses) was performed applying Principal Components Analysis (PCA) on the LIBS spectra to the potential LIBS calibration set spectra (with mean and center as the spectral preprocessing). Two PCs were enough to explain the whole variance (PC1: $81.51 \%$ and PC2: $11.02 \%$ ), and the addition of extra components did not increase significantly the explained data variance. Moreover, there was a high reproducibility in the measurement process of the calibration pellets. From the 57 measurements only six outliers were detected. The identification of these outliers was considered positive if the replicate measurements for each pellet were away from the other replicates.

Moreover, a suitable dataset (representative and reflecting the maximum variance shown by samples) to proceed to the quantification was confirmed by means of PCA (PC1: $71.31 \%$ and PC2: $13.93 \%$ ) applied to the potential LIBS spectra set. This included the calibration set, the terrestrial analogues to meteorites samples to analyze (LDG, DG and black steel slag) and the test set (pellets with mixture of the reference material and ethyl-cellulose). As it can be seen in the Fig. 3, sample dataset and the selected validation set could be in the same range of concentration that the calibration spectra set at the $99 \%$ confidence level.

Two different calibration approaches were used with the whole dataset: univariate calibration and multivariate calibration using Partial Least Squares (PLS).

\subsubsection{Univariate calibration}

Firstly, a univariate calibration using the UV wavelength region was carried out. The characteristics emission lines for $\mathrm{Al}, \mathrm{Ca}, \mathrm{Fe}, \mathrm{Mg}, \mathrm{Si}$, and $\mathrm{Ba}$ in the $\mathrm{UV}$ wavelength region of a LIBS spectrum of the calibration set can be seen in the Fig. 4.a. The determination of the peak areas of the LIBS spectra was studied without normalization and using normalization. The normalization consisted in calculating the sum of all the lines intensity values, and the chosen lines were corrected with respect to that value. These results were similar to those without normalization, therefore, their peak areas without normalization were considered for building the univariate models.

A spectral line without overlapping was selected for each element: $\mathrm{Al}(\mathrm{I}) 308.21 \mathrm{~nm}, \mathrm{Ca}$ (II) $396.87 \mathrm{~nm}, \mathrm{Fe}$ (I) $373.74 \mathrm{~nm}, \mathrm{Mg}$ (I) $285.16 \mathrm{~nm}, \mathrm{Si}(\mathrm{I}) 288.16 \mathrm{~nm}$ and $\mathrm{Ba}(\mathrm{II}) 389.14 \mathrm{~nm}$ (neutral (I) or single ionized species (II)). Na was not taken into account because it did not present strong or relevant lines in the UV spectral range according to the intensities given by our equipment.

Then, the areas of $\mathrm{Al}, \mathrm{Ca}, \mathrm{Fe}, \mathrm{Mg}, \mathrm{Si}$ and $\mathrm{Ba}$ selected lines were represented in front of the concentrations to obtain the univariate calibration curves. In all cases, univariate calibration models presented low linearity with correlation coefficient too low $\left(\mathrm{r}_{\text {cal }}<0.90\right)$, showing low 
robustness for the models. The poor linearity of the univariate calibration curves affects the accuracy and the precision of the prediction. Thus, the univariate calibration curves could be used only for rapid estimations of the elemental compositions in the samples but not for accurate estimation of unknown samples.

\subsubsection{Multivariate calibration}

PLS regression method type I (PLS1) was used as a single response variable (elemental concentration) versus the observable variable (spectra, intensity at each spectrometer channels). PLS multivariate models were created using the calibration data set, which predicted a limited number of output values (elemental concentrations) from a very large number of input variables (intensity at multiple wavelengths).

This calibration involved three phases, namely regression, validation and prediction. In the regression phase, the LIBS spectra of 19 calibration pellets (3 replicates in different sites of each pellet) were used to make the calibration data set. The whole set of data was used to construct the mathematical model per analyte. For the validation phase, it was employed a test set with LIBS spectra of six steelmaking slag RM pellets (3 replicates in different sites of each pellet) of different concentrations diluted with ethylcellulose. The final phase of calibration was the prediction, where the robustness of the model was tested with another set of samples that was completely independent from the calibration and validation data set; these were the LIBS spectra of the impact glasses and the steel slag samples.

It was compared the use of both spectral range (UV plus IR) together or separately in order to observe drawbacks or advantages in each case and with the aim of simplifying the method of analysis.

\subsubsection{Combined UV + IR LIBS spectra}

UV and IR spectral ranges were used jointly. The obtained LIBS spectra of the oxide materials revealed clear differences between calibration samples of different composition, as it can be seen in the Fig. 4.

Besides, on the basis of the spectral selectivity and sensitivity of our portable instrument, the following characteristic emission lines of each element (NIST database) were selected as the significant emission lines for the quantitative determination: $\mathrm{Ca}(\mathrm{I}) 616.12 \mathrm{~nm}, \mathrm{Fe}(\mathrm{I}) 275.01 \mathrm{~nm}$, $\mathrm{Fe}$ (I) $667.15 \mathrm{~nm}, \mathrm{Mg}$ (II) $279.63 \mathrm{~nm}, \mathrm{Na}$ (I) $589.16 \mathrm{~nm}, \mathrm{Ba}(\mathrm{I}) 705.74 \mathrm{~nm}$, and $\mathrm{Si}$ (II) $634.88 \mathrm{~nm}$.

Predictive models were designed with the aforementioned 51 final calibration samples. Over the calibration set spectra, several spectral ranges combined with different preprocessing algorithms were tested, and the optimum number of PLS factors with the minimum of the predicted residual error (RMSE) was selected. For that, the calibration set spectra was applied for the 
construction of around 450 preliminary PLS calibration models for each analyte (aprox. 3200 PLS models) using the full spectral range (UV-IR).

Ten preprocessing methods were employed: Constant Offset Elimination (COE), Straight Line Subtraction (SLS), Vector Normalization (SVN), Multiplicative Scatter Correction (MSC), first derivative (FD), Min-Max Normalization (MMN), Second Derivate remove constant background signals, First derivate (FD) and Straight line subtraction, First derivate (FD) and Vector normalization (SNV) and First derivative and MSC.

Furthermore, the mentioned spectral preprocessings were taken with only a spectral range of $+/$ $5 \mathrm{~nm}$ from the characteristic line intensity of each specific element to build up PLS models that reflected the elemental concentration, but there were unsatisfactory results. Hence, they were not taken into account.

The preliminary PLS models were used for selecting the final PLS models (one for each analyte) based on adequate spectral range (including the characteristics and strong emission lines of each analyte), high regression coefficient (r), appropriate number of PLS factors (rank) and the lowest relative errors of cross validation (RMSECV). In addition, the residual predictive deviation (RPD) and the Bias value were employed as quality indicators of prediction accuracy and predictive ability of the PLS models. Because of the high volume of results generated, the best PLS models of each analyte with their statistical parameters have been summarized in the Table 1.

From none to different spectral preprocessing methods were required as optimal spectral pretreatment, such as SNV and MSC as well as a combination of them (FD + MSC). The number of PLS factors (Rank) were low and ranged from 2 (for $\mathrm{Ba}$ ) to a maximum one of 10 (for Na) (see Table 1). The RPD values were around 2 (except in Na). The highest values of RDP suggest that the methods have a good prediction capability. Moreover, low values for bias were found (from 0.3 for $\mathrm{Ba}$ and $\mathrm{Ca}$, and 1.2 for $\mathrm{Al}$ ); the lower bias value, the better for the estimated unknowns.

Fig. 5 shows the comparison between the calibration and validation lines from the dataset in the case of $\mathrm{Al}, \mathrm{Fe}, \mathrm{Mg}$, and $\mathrm{Si}$. As it can be noticeable, there are not significant differences between the calibration and validation line, with $\mathrm{r}$ values between 0.8 (for $\mathrm{Fe}$ ) and 0.9 (for $\mathrm{Mg}$ ).

\subsubsection{UV versus IR LIBS spectra}

With the aim of minimizing analysis time and the number of spectral data to the statistical treatment, the same methodology of PLS calibration was applied separately to each spectral region. Table 2 and 3 summarize details of the spectral preprocessing, spectral range and quality parameters of the final PLS models selected for each analyte. 
In the case of PLS models of the LIBS spectra belonging to the UV range, the RPD values were higher (2.63 for $\mathrm{Mg}$ ) than in the case of those belonging to the IR range. It had also better regression coefficients (between 0.77 and 0.92), bias (low values, for instance 0.02 for $\mathrm{Fe}$ ) and RMSECV values (around 12\%). An explanation about the worst results with the IR spectral range could be due to distortions produced by self-absorption, lower number of emission characteristics lines, higher overlapping of the emission lines in that range, laser energy fluctuations, etc. Consequently, despite some of those parameters could improve using only the UV spectral range, the combination of both ranges (UV plus IR) offered high regression coefficients and RPD, low RMSECV and rank values, and besides, provided the possibility to obtain all the characteristics and strong emission lines of each analyte ( $\mathrm{Al}, \mathrm{Ba}, \mathrm{Ca}, \mathrm{Fe}, \mathrm{Mg}, \mathrm{Na}$, and $\mathrm{Si}$ ), not given in each separate spectral range.

According to that, it was considered to use the PLS models obtained when we combined UV and IR spectral range for the next steps of the method development.

\subsubsection{Validation of PLS models using all the spectral range (UV+IR)}

With the final PLS models for each analyte ( $\mathrm{Ca}, \mathrm{Al}, \mathrm{Fe}, \mathrm{Si}, \mathrm{Na}, \mathrm{Mg}$, and $\mathrm{Ba}$ ), two different types of validation were performed: cross-validation (LOO) and test set validation.

Firstly, the constructed PLS calibration models for each analyte were validated using leave-oneout (LOO) cross validation, in which each sample in the calibration set is successively left out and treated as an unknown while the model is trained. In our case of 51 measurements, each new model was made on 50 measurements. The estimation of RMSE of cross-validation is RMSECV and the values obtained can be seen in the Table 1. The PLS models had low RMSECV (around 15\%) taking into consideration the high concentration values of each analyte used in the calibration data set.

Finally, the models were validated using an external set of samples (test set validation). For that purpose, we selected the steelmaking slag reference material, whose composition is a mixture of oxides as in our calibration set (oxide pellets). The 6 pellets of this RM with different concentrations of the studied elements were measured. The test set validation gave excellent results mainly to $\mathrm{Fe}, \mathrm{Mg}$, and $\mathrm{Si}$, and satisfactory for $\mathrm{Al}$ and $\mathrm{Ca}$. The $\mathrm{RM}$ has not certificated values for $\mathrm{Ba}$ and $\mathrm{Na}$.

\subsection{LIBS and $\mu$ - XRF analysis of the weathered terrestrial analogues to meteorites}

As it is mentioned above, the predictive ability of the PLS models was evaluated on the LIBS spectra of the impact glasses (see Fig. 6) and the black steel slag samples. The statistic index of Mahalanobis distance warned us about the applicability of the PLS models to a new sample. Thus, if the samples used in the calibration data set are similar to these new predicted samples, 
the value of this distance is low [31]. Our values of Mahalanobis distance were low $(<1$ in all the cases), except for $\mathrm{Al}$ and $\mathrm{Ca}$ in a few LDGs and DGs. Then, the predicted concentrations were not considered as outliers by the selected PLS models.

Furthermore, comparing our elemental concentration values (in terms of their oxide values) with others also obtained in steel slags with laboratory LIBS instrumentation [32], it could be observed that the same concentration range was obtained in both cases (see Fig. S1). The range of predicted concentrations of the impact glasses (LDG and DG) was also in accordance with the results of other authors in the bibliography [33-36].

Moreover, the predicted concentration values of $\mathrm{Ba}, \mathrm{Ca}, \mathrm{Fe}, \mathrm{Mg}, \mathrm{Na}$ and $\mathrm{Si}$ for both types of weathered terrestrial analogues obtained by the selected PLS model were in concordance with the composition tendency found by $\mu-\mathrm{XRF}$, taking into account the heterogeneity of the analyzed samples (see Fig. 7), except for Al.

\section{Conclusions}

A fast, cheap and effective quantitative methodology has been developed for in situ analysis of $\mathrm{Si}, \mathrm{Al}, \mathrm{Mg}, \mathrm{Ca}, \mathrm{Ba}, \mathrm{Na}$, and $\mathrm{Fe}$ in weathered terrestrial analogues to meteorites by means of portable LIBS using multivariate calibration line approach. To guarantee the homogeneity of the powdered pellets of the standards, the base of this work, the elemental and molecular distribution was always checked at the size of the LIBS spot (around $200 \mu \mathrm{m}$ ) through SEMEDS and Raman imaging.

Univariate calibration, the most common approach used in LIBS, did not provide satisfactory results for the quantitative analysis of this kind of samples. Irrespective of the selected emission lines for the different elements, the calibration models presented low linearity and correlation coefficient values. Hence, multivariate calibration was used to quantify $\mathrm{Fe}, \mathrm{Mg}, \mathrm{Si}, \mathrm{Al}, \mathrm{Ba}, \mathrm{Na}$, and $\mathrm{Ca}$ using UV and NIR spectral ranges. After a comparison between using these spectral ranges together and using them separately, a much more robust calibration approach was obtained by considering multivariate models with both UV and IR ranges. The selected PLS models for the seven elements showed linearity in the calibration and validation curve with the selected dataset. The capability of this approach was checked using slag reference material with excellent results in the case of $\mathrm{Fe}, \mathrm{Mg}$, and $\mathrm{Si}$, and satisfactory for $\mathrm{Al}$ and $\mathrm{Ca}$. Moreover, concentration values of $\mathrm{Fe}, \mathrm{Mg}, \mathrm{Si}, \mathrm{Al}, \mathrm{Ba}, \mathrm{Na}$ and $\mathrm{Ca}$ of impact glasses and steel slag samples were calculated, giving similar results to those by $\mu$-XRF.

We concluded that the PLS approach provided better predictions than the univariate calibration for these type of silicate based materials, which have been subjected to high temperatures during their formation. This finding is relevant to develop calibration strategies to analyse other types 
of silicate materials formed at high temperatures, like silicate based meteorites and other kind of extraterrestrial materials.

\section{Acknowledgements}

This work has been financially supported through the projects "Development of the Raman instrument for the ESA Mission Exomars2018: Science support, equipment testing and operation support" (Grant ESP2014-56138-C3-2-R), funded by the Spanish Ministry of Economy and Competitiveness (MINECO) and the European Regional Development Fund (FEDER) and "Analytical approach to the study of Planetary Geochemical Processes" (Grant PES15-09), funded by the University of the Basque Country (UPV/EHU). Technical and human support provided by the Raman-LASPEA Laboratory and the X-Ray Service: Unit of rocks and minerals of the SGIker (UPV/EHU, MICINN, GV/EJ, ERDF and ESF) is gratefully acknowledged.

\section{References}

[1] G.S. Senesi, Laser-Induced Breakdown Spectroscopy (LIBS) applied to terrestrial and extraterrestrial analogue geomaterials with emphasis to minerals and rocks, Earth-Sci. Rev. 139 (2014) 231-267.

[2] J. Aramendia, L. Gomez-Nubla, K. Castro, S. Fdez-Ortiz de Vallejuelo, G. Arana, M. Maguregui, V.G. Baonza, J. Medina, F. Rull, J.M. Madariaga, Overview of the techniques used for the study of non-terrestrial bodies: Proposition of novel non-destructive methodology, TrAC 98 (2018) 36-46.

[3] M. Hornackova, J. Plavcan, J. Rakovsky, V. Porubcan, D. Ozdin, P. Veis, Calibration-free laser induced breakdown spectroscopy as an alternative method for found meteorite fragments analysis , Eur. Phys. J. Appl. Phys. 66 (2014) 10702/1-10702/10.

[4] J. Kovacs, I. Sajo, Z. Marton, V. Jager, T. Hegedus, T. Berecz, T. Toth, P. Gyenizse, A. Podobni, Andras, Csatalja, the largest H4-5 chondrite from Hungary, Planet. Space Sci. 105 (2015) 94-100.

[5] D. Ozdin, J. Plavcan, M. Hornackova, P. Uher, V. Porubcan, P. Veis, J. Rakovsky, J. Toth, P. Konecny, J. Svoren, Mineralogy, petrography, geochemistry, and classification of the Kosice meteorite, Meteorit. Planet. Sci. 50 (2015) 864-879.

[6] J.R. Thompson, R.C. Wiens, J.E. Barefield, D.T. Vaniman, H.E. Newsom, S.M. Clegg, Remote Laser Induced Breakdown Spectroscopy (LIBS) Analyses of DaG 476 and Zagami Martian Meteorites. J. Geophys. Res. 111 (2006) 6:1-6:9.

[7] A. De Giacomo, M. Dell'Aglio, O. De Pascale, S. Longo, M. Capitelli, Laser Induced Breakdown Spectroscopy on Meteorites. Spectrochim. Acta B, 62 (2007) 1606-1611. 
[8] M. Dell'Aglio, A. De Giacomo, R. Gaudiuso, O. De Pascale, G.S. Senesi, S. Longo, LIBS Applications to Meteorites: Chemical Analysis and Composition Profiles. Geochim. Cosmochim. Acta, 74 (2010) 7329-7339.

[9] R. Gaudiuso, M. Dell'Aglio, O. De Pascale, G.S. Senesi, A. De Giacomo, Laser Induced Breakdown Spectroscopy for Elemental Analysis in Environmental, Cultural Heritage and Space Applications: A Review of Methods and Results, Sensors 10 (2010) 7434-7468.

[10] J. Rakovský, P. Čermák, O.Musset, P. Veis, A review of the development of portable laser induced breakdown spectroscopy and its applications, Spectrochem. Acta Part B, 101 (2014) 269-287.

[11] S. Fdez-Ortiz de Vallejuelo, A. Gredilla, L. Gomez-Nubla, E. Ruiz-Romera, A. Zabaleta, J. M. Madariaga. Portable laser induced breakdown spectrometry to characterize the environmental impact of potentially hazardous elements of suspended particulate matter transported during a storm event in an urban river catchment. Microchem. J. 135 (2017) 171179.

[12] D. W. Hahn and N. Omenetto, Laser-induced breakdown spectroscopy (LIBS), Part I: Review of basic diagnostics and plasma particle interactions: Still-challenging issues within the analytical plasma community, Appl. Spectrosc. 64 (2010) 335A-366A.

[13] D. W. Hahn and N. Omenetto, Laser-induced breakdown spectroscopy (LIBS), Part II: Review of instrumental and methodological approaches to material analysis and applications, Appl. Spectrosc. 66 (2012) 347-419.

[14] S. Wu, T. Zhang, H. Tang, K. Wang, X. Yang, H. Li, Quantitative analysis of nonmetal elements in steel using laser-induced breakdown spectroscopy combined with random forest, Anal. Methods. 7 (2015) 2425-2432.

[15] V.K .Unnikrishnan, K. Mridul, R. Nayak , K.Alti, V.B. Kartha, C. Santhosh, G.P. Gupta, B.M. Suri, Calibration-free laser-induced breakdown spectroscopy for quantitative elemental analysis of materials, Pramana- J. Phys. 79 (2012) 299-310.

[16] F.J. Fortes, J. Moros, P. Lucena, L.M. Cabalín, J.J. Laserna, Laser Induced Breakdown Spectroscopy, Anal. Chem. 85 (2013) 640-669.

[17] J.W. Batista Braga, L.C. Trevizan, L.C. Nunes, I. Aparecida Rufini, D. Santos Jr., F.J. Krug, Comparison of univariate and multivariate calibration for the determination of micronutrients in pellets of plant materials by laser induced breakdown spectrometry, Spectrochim. Acta B 65 (2010) 66-74.

[18] M.D. Dyar, M.L. Carmosino, E.A. Breves, M.V. Ozanne, S.M. Clegg, R.C. Wiens, Comparison of partial least squares and lasso regression techniques as applied to laser-induced breakdown spectroscopy of geological samples, Spectrochim. Acta B 70 (2012) 51-67. 
[19] J.M. Andrade, G. Cristoforetti, S. Legnaioli, G. Lorenzetti, V. Palleschi, A.A. Shaltout, Classical univariate calibration and partial least squares for quantitative analysis of brass samples by laser-induced breakdown spectroscopy, Spectrochim. Acta B 65 (2010) 658-663.

[20] J.M. Tucker, M.D. Dyar, M.W. Schaefer, S.M. Clegg, R.C. Wiens, Optimization of laserinduced breakdown spectroscopy for rapid geochemical analysis, Chem. Geol. 277 (2010) 137148.

[21] B. C. M. Butler, Al-rich pyroxene and melilite in a blast-furnace slag and a comparison with the Allende meteorite, Mineral. Mag. 41 (1977) 493-499.

[22] C. Wert, M. Weller, Internal friction of the glassy tektites, J. Alloys Compd 310 (2000) 5458 .

[23] J. Kempl, P.Z. Vroon, E. Zinngrebe, W. van Westrenen, Si isotope fractionation between $\mathrm{Si}$-poor metal and silicate melt at pressure-temperature conditions relevant to metal segregation in small planetary bodies, Earth Planet. Sci. Lett. 368 (2013) 61-68.

[24] J. Garcia-Guinea, V. Correcher, L. Recio-Vazquez, E. Crespo-Feo, R. Gonzalez-Marti, L. Tormo, Influence of accumulation of heaps of steel slag on the environment: determination of heavy metals content in the soils, Annals of the Brazilian Academy of Sciences 82 (2010) 267277.

[25] L. Gomez-Nubla, J. Aramendia, S. Fdez-Ortiz de Vallejuelo, K. Castro, J.M. Madariaga. From Portable to SCA Raman devices to characterize harmful compounds contained in used black slag produced in Electric Arc Furnace of steel industry, J Raman Spectrosc., 44 (2013) 1163-1171.

[26] L. Gomez-Nubla, J. Aramendia, S. Fdez-Ortiz de Vallejuelo, A. Alonso-Olazabal, K. Castro, M,C. Zuluaga, L.A. Ortega, X, Murelaga, J.M. Madariaga, Multispectroscopic methodology to study Libyan Desert Glass and its formation conditions. Anal Bioanal Chem. 409 (2017) 3597-3610.

[27] L. Gomez-Nubla, J. Aramendia, A. Alonso-Olazabal, S. Fdez-Ortiz de Vallejuelo, K. Castro, M.C. Zuloaga, L.A. Ortega, X. Murelaga, J.M. Madariaga. Darwin impact glass study by Raman spectroscopy in combination with other spectroscopic techniques. J. Raman Spectrosc. 46 (2015) 913-919.

[28] I. Torre-Fdez, J. Aramendia, L. Gomez-Nubla, K. Castro, J. M. Madariaga, Geochemical study of the Northwest Africa 6148 Martian meteorite and its terrestrial weathering processes, J. Raman. Spectrosc. DOI 10.1002/jrs.5148.

[29] E. Dubina, L. Korat, L. Black, J. Strupi-Šuput, J. Plank, Influence of water vapour and carbon dioxide on free lime during storage at $80{ }^{\circ} \mathrm{C}$, studied by Raman spectroscopy, Spectrochim. Acta A 111 (2013) 299-303. 
[30] Q. Sun, The Raman OH stretching bands of liquid water, Vib. Spectrosc. 51 (2009) 213217.

[31] R. De Maesschalck, D. Jouan-Rimbaud, D.L. Massart, The Mahalanobis distance, Chemometr. Intell. Lab. 50 (2000) 1-18.

[32] B. Praher, R. Rossler, E. Arenholz, J. Heitz, J.D. Pedarning, Quantitative determination of element concentrations in industrial oxide materials by laser-induced breakdown spectroscopy, Anal. Bioana. Chem. 400 (2011) 3367-3375.

[33] A. Longinelli, G. Sighinolfi, V. de Michele, E. Selmo, d180 and chemical composition of Libyan Desert Glass, country rocks, and sands: New considerations on target material, Meteorit. Planet. Sci. 46 (2011) 218-227.

[34] A. Greshake, C. Koeberl, J. Fritz, W. Reimold, Brownish inclusions and dark streaks in Libyan Desert Glass: evidence for high-temperature melting of the target rock. Meteorit. Planet. Sci. 45 (2010) 973-989.

[35] G. Volksch, K. Heide, Structure and crystallization in natural glasses. Proceedings of International Congress on Glass, 18th, San Francisco, United States, 1998, 2124-2128.

[36] K.T. Howard, Geochemistry of Darwin glass and target rocks from Darwin crater, Tasmania, Australia. Meteorit. Planet. Sci. 43 (2008) 479-496. 


\section{Figure captions}

Fig.1. Concentrations of the elements ( $\mathrm{Ba}, \mathrm{Na}, \mathrm{Ca}, \mathrm{Al}, \mathrm{Fe}, \mathrm{Si}$, and $\mathrm{Mg}$ ) present in the powder pellets belonging to the calibration curve (1-19). The concentrations are given in $\%$ weight.

Fig.2. $i$. SEM image of a calibration sample; ii. SEM mapping of a calibration sample. Green: $\mathrm{Ca}$, blue: Fe, reddish: $\mathrm{Mg}$, white: Ba, khaki: Si and brownish-reddish: Al. Na was not detected probably because this sample had the lowest sodium concentration; iii. Raman microscopic image (a) and distribution of $\mathrm{Na}_{2} \mathrm{O}+\mathrm{Na}_{2} \mathrm{O}_{2}$ (b), $\mathrm{SiO}$ (c) and $\mathrm{Fe}_{2} \mathrm{O}_{3}$ (d) in a coloured area of $70 \times 70 \mu \mathrm{m}$ for one of the calibration samples.

Fig.3. Projection of the scores on the space formed by the first and second PCs of LIBS data set spectra in the range of UV and IR. Orange: test set (RM), Black: calibration set, Green: Darwin Glass, Blue: Libyan Desert Glass, and Pink: black steel slag.

Fig.4. Six representative LIBS spectra of $7,10,12,13,16$, and 17 calibration pellets with significant difference in the content of oxide weight: (a) UV and IR (b) spectral ranges.

Fig.5. Model and validation plots produced with PLS1. It is displayed the known versus PLS predicted concentrations of $\mathrm{Al}, \mathrm{Fe}, \mathrm{Mg}$, and $\mathrm{Si}$ in $\mathrm{mg} \cdot \mathrm{Kg}^{-1}$. The green circles and solid line represent the calibration curve, and the blue line represents the validation curve. The red circles are outliers.

Fig.6. Some representative LIBS spectra measurements on LDG and DG samples with the characteristic emission lines of $\mathrm{Al}, \mathrm{Fe}, \mathrm{Mg}, \mathrm{Na}, \mathrm{Ca}, \mathrm{Si}$, and $\mathrm{Ba}$ in the $\mathrm{UV}$ and NIR region.

Fig. 7. Maximum and minimum concentration values (\%) of LDG, DG and the three slag samples obtained by LIBS analysis (black) and $\mu-\mathrm{XRF}$ (green). N.D.: not detected.

Fig. S1. Maximum, minimum and mean concentration values (\%) of our common steel slag (blue) and those of other work (red). 